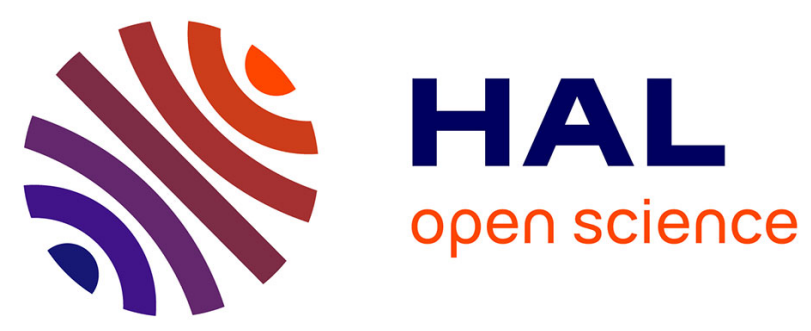

\title{
MULTI-AGENT PLANNING FOR THE ROBOCUP RESCUE SIMULATION Applying Clustering into Task Allocation and Coordination
}

Amr Hussein, Carmen Gervet, Slim Abdennadher

\section{- To cite this version:}

Amr Hussein, Carmen Gervet, Slim Abdennadher. MULTI-AGENT PLANNING FOR THE ROBOCUP RESCUE SIMULATION Applying Clustering into Task Allocation and Coordination. ICAART, 2012, Lisbon, Portugal. hal-01742393

\section{HAL Id: hal-01742393 \\ https://hal.umontpellier.fr/hal-01742393}

Submitted on 24 Mar 2018

HAL is a multi-disciplinary open access archive for the deposit and dissemination of scientific research documents, whether they are published or not. The documents may come from teaching and research institutions in France or abroad, or from public or private research centers.
L'archive ouverte pluridisciplinaire HAL, est destinée au dépôt et à la diffusion de documents scientifiques de niveau recherche, publiés ou non, émanant des établissements d'enseignement et de recherche français ou étrangers, des laboratoires publics ou privés. 


\title{
MULTI-AGENT PLANNING FOR THE ROBOCUP RESCUE SIMULATION Applying Clustering into Task Allocation and Coordination
}

\author{
Amr Hussein, Carmen Gervet, and Slim Abdennadher \\ Faculty of Media Engineering and Technology, German University in Cairo, Egypt \\ \{amr.labib-hussein, carmen.gervet, slim.abdennadher $@ @$ guc.edu.eg
}

Keywords: Multi-Agent Planning, Clustering, RoboCup, Rescue, RoboCup Rescue Simulation.

Abstract: The RoboCup Rescue Simulation system provides a rich environment for developing novel techniques for multi-agent systems. The simulation provides a city map modeled as buildings and roads with civilians amongst them. A disaster scenario is simulated causing buildings to catch fire, roads to get blocked, and civilians to get injured and/or buried. The main goal is to use the available emergency services (rescue agents) to extinguish the fires, clear the roads, and rescue the civilians. This paper describes a new multi-agent planning approach applied to the RoboCup Rescue problem. Task allocation coordination is done through clustering the map into several overlapping maps each with a different group of agents assigned to it. Our results show that by applying this approach to the RoboCup Rescue Simulation we did compete with the top teams in the 2011 RoboCup Rescue Agent Simulation Competition and ranked 3rd in our first participation in the competition.

\section{INTRODUCTION}

The RoboCup Rescue project, established in 2001, aims at promoting research and development in the rescue domain at various levels, involving multi-agent team work coordination, physical robotic agents for search and rescue, information infrastructures, personal digital assistants, a standard simulator and decision support system, evaluation benchmarks for rescue strategies, and robotic systems that should all be integrated into a comprehensive system in the future (Kitano and Tadokoro, 2001; Skinner and Ramchurn, 2010).

In the RoboCup Rescue Simulation a model of an earthquake in an urban center is simulated and teams compete to produce efficient response policies for the simulated emergency services. The earthquake model covers building collapse, roads blocked by rubble and other debris, traffic movement, fire, and injuries to civilians and emergency services workers.

The simulated world consists of buildings, roads, and emergency services. Buildings can catch fires and collapse. Some buildings are refuges and can be used to heal injured civilians or refill fire brigades, Roads include traffic movement and blocked roads. Emergency services include fire brigades, ambulance teams, and police forces.

The target of the simulation is to find the optimal online strategy that best utilizes the emergency services to save the maximum number of lives, extinguish the maximum number of buildings, and clear the maximum number of blockades.

The RoboCup Rescue Simulation defines a multiagent planning optimization problem that requires an efficient strategy for distributing up-to 30 agents over the simulated map, an efficient strategy for traversing the map and discovering the unknown emergency events, and efficient communication to share information among the agents.

The German University in Cairo team RMAS_ArtSapience participated in the 2011 RoboCup Rescue Agent Simulation Competition in Istanbul, Turkey. The work described in this paper and our results have led us to qualify for our first participation and win the third place in the competition.

\subsection{Related Work}

The approaches discussed in this section are all based on the team description papers that belong to the teams (excluding ours) that participated in the final round of the 2011 RoboCup Rescue Competition.

- SEU_RedSun, Southeast University, China Ranked first in the 2011 competition and partic- 
ipating since 2008, SEU_RedSun's approach depended on accurate world modeling via multicommunication. Tasks were assigned via a centralized decision support system. Particle filters were used to predict fire spread. Their main strength lied in the ability to predict fires early and good utilization of agent communication. Their main weakness lied on depending on the availability of communication and not having a well defined strategy for communication-less scenarios.

\section{- Poseidon, Farazanegan High School, Iran}

Ranking second in the 2011 competition and participating since 2009, Poseidon's approach depended on discovering connected and unconnected parts of the world via agent communication to build an enhanced world model. The used off-line precomputed schedules optimized using genetic algorithms. Their approach also depending on dividing the map evenly. Their main strength lies in the optimized schedules and the enhanced world model. Their weakness was in dividing the map evenly without taking into consideration the map structure or distribution of buildings and in relying on the availability of communication.

- IAMRescue, University of Southampton, UK Ranking fourth in the 2011 competition and participating since 2008, IAMRescue's approach a hierarchical decision-making system supported by disaster prediction via learning fire spread. Their decision-making system also depends on agent communication for coordination. The accuracy of the decision-making system marked IAMRescue's main strength point, but similar to the previous approaches, the lack of communication-less strategies was weakness in their approach.

\subsection{Our Contribution}

In this paper we show a novel approach of modeling the rescue problem as a multi-agent planning problem. Our approach to solve the different challenges and produce competitive results is based on the following contributions:

- Use of clustering, instead of dividing the map evenly, to divide the map into regions. This will then allow us to distribute the agents over these regions and perform the rescue operations within them.

- A multi-agent communication model was created for agents to exchange information and ask for help.

\section{OUR APPROACH}

We choose to solve the rescue problem by modeling it as a multi-agent planning problem (Weerdt et al., 2005). The main reason behind choosing to model the rescue problem as a multi-agent planning problem is the ease of task definition and division. The nature of the presented rescue problem provides well defined tasks that can be easily divided among the different types of agents with a main goal of performing and optimizing the rescue process. The structure explained The following section explain how the different phases of multi-agent planning are created and how they contribute to solve the rescue problem.

\subsection{Defining Tasks}

The target of this phase is to refine the global tasks and divide it into smaller individual tasks. The rescue problem can be divided into three main tasks: extinguishing fires, saving civilians, and clearing blocked roads. The previous tasks could be furthermore divided into several individual tasks. Figure 1 shows a how the rescue tasks are divided into individual tasks.

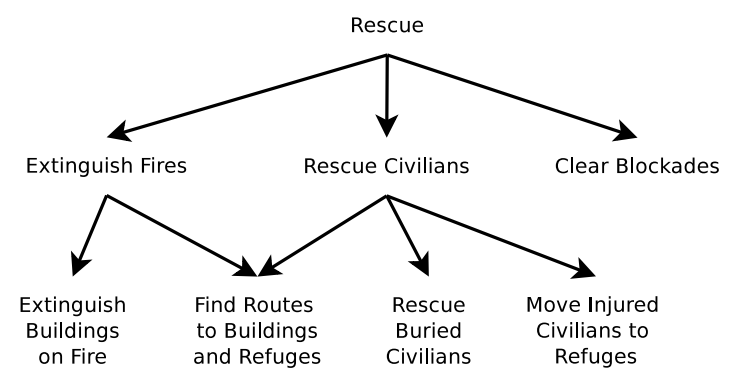

Figure 1: Rescue Tasks Tree Structure.

Finding routes to buildings and refuges will be done individually by all agents. Extinguishing buildings can only be done by fire brigades. Rescuing buried civilians and moving the injured ones can only be done by the ambulance teams. Clearing blockades can only be done by police forces.

\subsection{Task Allocation}

Task allocation is required to distribute and assign tasks to the agents. In our case, task definition already provided what each agent will be doing but did not specify where in the map it will carry out its action. Since it is impossible for each agent to timely traverse the whole map, the map was divided on the number of agents available. Previous approaches used to divide the map evenly into a grid which did not take into 
consideration the map structure and the distribution of the buildings in the map. In our approach, we choose to cluster the map on the buildings such that we can output regions of almost evenly distributed buildings. Each region will be assigned an agents that will traverse all of the buildings and roads in the region to search for events that require rescue actions.

This introduced a new challenge to the rescue agents due to blockades. A series of blocked roads can divide a single region into disconnected parts prohibiting, or delaying in the case of police forces, other agents from reaching some parts in the region. This challenge was solved in our approach with the use of fuzzy c-means (FCM) clustering (Bezdek, 1981; Bezdek et al., 1984). Unlike many of the clustering algorithms that produce disjoint non-empty clusters, such as K-means, FCM clustering algorithm has the ability to assign a data point to several clusters with a specific membership function. This allows some degree of overlap between the clusters.

Since FCM clustering produces intersecting clusters, the produced regions will have common buildings and roads. This will allow some of the buildings and roads within each region to be visited by at least two agents. If it happens that the area of intersection is disconnected in one cluster, it could be reachable from another cluster it belongs to. This will increase the chance that agents will be able to visit all buildings and roads in proper time.

\subsection{Coordination Before Planning}

Coordination is required before planing to define the rules and constraints that must be applied on planning to guarantee the satisfaction of the main goal, which is performing and optimizing the rescue process in our case. The approach used in task allocation included coordination. Dividing the map into regions provided constraints on where the agents will perform the rescue process. Assigning agents to regions provided constraints on which agents will perform the rescue process in which region.

Another coordination challenge presented itself when it came to optimizing the rescue process. In many cases, it was noticed that blockades can cause some agents to be initially stuck and some refuges unreachable. An agent is considered to be stuck when it is surrounded by blockades from all directions and cannot move. A refuge is considered to be unreachable if all roads leading to it are blocked. The approach used in task allocation and coordination depends on dividing the map and distributing the agents on the regions. Since blockades are initially unknown, stuck agents will be considered in this distribution, which has a big negative effect on the rescue performance, especially for regions with a small number of agents. On the other hand, refuges are used for treating injured civilian and refilling fire brigade tanks and they are the only buildings that do not catch fire. Blocked refuges will prevent the rescue process from being complete and also has a big negative effect on the rescue performance.

Since police forces are the only agents capable of clearing blockades, they are the only ones that do not get stuck. Normally, police forces are assigned to regions as discussed earlier. The approach devised in this paper gives the police forces the task of freeing all stuck agents and unreachable refuges before performing rescue processes in their assigned regions. Police forces are only aware of the initial location of all agents and refuges, but they are not aware of which of them are stuck or unreachable. The following approach explains how this problem is tackled:

- Initial agent and refuge locations are retrieved.

- FCM clustering is used to divide the locations into regions.

- A police force agent is assigned to each region.

- A constraint is added on planning for the police force agents that requires police forces to clear the roads to the location of the agents and refuges in the assigned regions produced here.

\subsection{Planning}

The planning phase involves the creation of the individual plans created for each agent putting in mind the global goal. In our case, planning is carried through considering the actions required to carry out the individual tasks the agents should perform and the constraints added in the coordination step. Each agent creates its own individual plan taking into consideration its type (police, fire, or ambulance agent) and the cluster the agent is assigned to. All agents will follow the routes that passes by all roads/buildings in their regions. We show below how we created the individual plans for each agent.

\subsubsection{Ambulance Teams}

Ambulance teams are assigned the task of rescuing buried civilians and moving injured civilians to refuges. As each agent follows its route, it will keep scanning the buildings for civilians. When buried civilians are found, the agent will attempt to rescue the civilian. If the agent finds a an injured civilian that needs to be transported to a refuge, it will load the civilian, move to the nearest reachable refuge, unload 
the injured civilian inside the refuge, and return back to the last location the agent checked. Each agent will keep track of unvisited buildings. Once a building is visited, it is removed from this list. If a building has several buried civilians, the agent will keep track of this building to give it higher priority in the rescue operation. If all buildings are visited and no more civilians need to be rescued, the ambulance team does not have to do any more work in its region.

\subsubsection{Fire Brigades}

Fire brigades will follow the same plan as the ambulance teams but they will not need to enter the buildings. Each agent will scan all the buildings in its route and check for fires. Once a fire is found, the agent begins extinguishing it. When it runs out of water, the agent goes to the nearest possible refuge to refill, keeping in mind any location it has passed that was on fire. Fires do not necessarily start right after the occurrence of the disaster; they could start anytime. Even extinguished buildings could ignite again. As a consequence, searching for fires does not stop and the fire brigades will continuously keep searching for fires.

\subsubsection{Police Forces}

Police forces also follow the same plan as the ambulance teams and the fire brigades, but, with an extra first step. Police forces start with clustering the initial location of all ambulance teams, fire brigades, and refuges. Each police is assigned to a region and moves to it to clear the roads to all agents and refuges in this region.

After the each police force is done with clearing the roads to all agents and refuges in it is assigned, it moves to the region it should be clearing the roads in. Police forces will respond to query messages sent by stuck agents in their regions. If an agent $\mathrm{s}$ region has no more blockades, the agent can move to another region and/or respond to query messages in different regions.

\subsection{Coordination after Planning and Execution}

Coordination after planning was carried out dynamically during the execution of the agents' plans. It mainly consisted of utilizing multi-agent communication and adding a criteria to have agents change it's region.

\subsubsection{Communication}

Communication was mainly used to exchange information on the rescue events that require attention. Communication messages were divided into informative and query messages. Informative messages were intended to inform other agents of sensed fires, blockades, and buried civilians. Informative messages are sent by agents that detect events they cannot act up on. On the other hand, query messages were intended to ask for help from agents of the same type as the sender. Query messages are sent when an agent realizes that it cannot perform a rescue action on it's own.

\subsubsection{Changing Region}

An agent can change its assigned region and carry out the rescue operation in the new one. Changing regions is only allowed in one of two cases: changing region temporarily in response to a communication message informing of an event in a different region or when in a agent does not find any rescue events in its region. In the first case the change is temporary and once the agent finishes response to the event, it returns back to its region.

In the second case, the agent will change its region if there are no more events that it can handle; the ambulance team would have visited all buildings and rescued all civilians, the fire brigade would have extinguished all the fires, and the police forces would have cleared all roads. If some regions are overloaded with rescue events and the agents assigned to them were unable to finish these events, none of them will change regions. On the other hand, agents in other regions that are either event free or with handled events will change keep changing regions until they reach the overloaded region. This will have the agents accumulate in overloaded regions and cooperate in performing the rescue operation faster.

\section{EVALUATION}

The main performance measure used to judge the efficiency of the implemented approach was the final simulation score of the rescue simulation. The score is automatically calculated by the simulation kernel and a score chart is produced to show the different parameters that contributed to the final score. The full detailed scores of the 2011 competition can found on the RoboCup Rescue Simulation (http: //roborescue. sourceforge. net/). 


\subsection{Results and Ranking}

Our team, RMAS_ArtSapience reached the final round of the 2011 competition and ranked third after tying on the 2 nd place in total ranking but lost to score difference. Our evaluation is based on two types of maps: maps with communication enables and communication-less maps.

\subsubsection{Map with Communication}

The maps with agent communication enabled are designed to test the ability of the agents to detect the fires early int he simulation and coordinate with other agents through communication to extinguish the fires and clear the blocked roads around the fires. Our team ranked in most cases in the top 4 in these maps. Table (TO BE ADDED) shows the results in some chosen samples from the 2011 competition. The main strength of our approach is the efficient agent distribution. Police forces where able to spread through the map and clear most of the blockades fast enough during the simulation.

\subsubsection{Communication-less Maps}

The communication-less maps did not allow the use of multi-agent communication. This made it hard for the agents to discover the location of fires. In addition to that, fires started randomly during the simulation, which furthermore increased the fire discovery challenge. The scenario is designed to mainly test how agents will perform in the absence of communication and how it affects their rescue actions.

Our approach was able to control the fires more efficiently than the other teams. This was due to efficient distribution of the agents over the map achieved through the use of clustering without depending on agent communication. The final scores, as seen in table (TO BE ADDED), showed that in some cases our team achieved a significantly higher scores than all other teams competing in the map.

\section{CONCLUSION}

The RoboCup Rescue Agent Simulation provided an interesting set of challenges for multi-agent systems and planning. The rescue problem was modeled into a multi-agent planning problem and it was possible to build an agent structure capable of solving this problem.

The multi-agent planning problem consisted of mainly two components, coordination and planning.
Coordination was carried out through the use of clustering, namely the fuzzy c-means clustering technique, to divide the map into regions. Clustering provided an efficient approach of distributing the agents over the map. Planning was created by developing individual strategies for each type of agent. Supported with multi-agent communication, it was possible to add more coordination among the agents during the execution of their plans.

The approach used in this paper has contributed to qualifying to the 2011 RoboCup Rescue Agent Simulation competition and achieved the third place in the final round.

\section{REFERENCES}

Bezdek, J. C. (1981). Pattern Recognition with Fuzzy Objective Function Algorithms. Kluwer Academic Publishers, Norwell, MA, USA.

Bezdek, J. C., Ehrlich, R., and Full, W. (1984). Fcm: The fuzzy c-means clustering algorithm. Computers \& Geosciences, 10(2-3):191-203.

Kitano, H. and Tadokoro, S. (2001). RoboCup Rescue: A Grand Challenge for Multiagent and Intelligent Systems. AI Magazine, 22(1).

Skinner, C. and Ramchurn, S. (2010). The RoboCup Rescue Simulation Platform. In Proceedings of the 9th International Conference on Autonomous Agents and Multiagent Systems: volume 1 - Volume 1, AAMAS '10, pages 1647-1648, Richland, SC. International Foundation for Autonomous Agents and Multiagent Systems.

Weerdt, M. D., Mors, A. T., and Witteveen, C. (2005). Multi-agent planning: An introduction to planning and coordination. Technical report, In: Handouts of the European Agent Summer. 